\title{
La qualité de l'enseignement universitaire: Essai d'approche systematique*
}

\author{
YGAL LEIBU**
}

\section{RESUME}

A force de l'invoquer à tort et à travers, à force de l'utiliser pour justifier les actions les plus contradictoires dans les contextes les plus divers, la notion de qualité de l'enseignement s'est progressivement galvaudée, à l'instar de tant d'autres concepts surchargés de signification. Si bien qu'aujourdhui on ne peut retirer de cette notion que ce qu'on y met au départ. En d'autres termes, il semble nécessaire, pour redonner un sens univoque à cette notion, de la dégager d'abord de la gangue d'artifices et de parti-pris qui l'entourent, que ce soit ouvertement ou par omission. Cela revient à dire qu'il faut aborder cette notion complexe de façon à couvrir la majeure partie sinon la totalité des aspects qu'elle implique. C'est là l'objectif premier du texte. D'autre part, on se propose également d'y montrer qu'il y a place, dans les universités, au-delà de pétitions de principe et d'affirmations impossibles à prouver, pour la mise en oeuvre de processus tangibles d'appréciation de la qualité des activités d'enseignements, ainsi que des programmes d'études courants ou nouveaux.

Une ébauche rapide de l'évolution du système universitaire au Québec et un aperçu de la conjoncture actuelle mettront en relief la nécessité d'agir en vue d'améliorer la qualité de l'enseignement universitaire.

Le chapitre suivant exposera les principales difficultés conceptuelles, analytiques et opératoires auxquelles se heurtent les actions visant à évaluer la qualité de l'enseignement. On en conclut que la qualité est un jugement de valeur, qu'il faut l'aborder en termes de relativité. Autrement dit, on en conclut que, pour apprécier la qualité ainsi que pour estimer si elle s'améliore - objectif ultime de l'entreprise, il faut se reporter à des standards qui peuvent varier eux-aussi dans le temps, selon les objectifs poursuivis.

Au chapitre suivant on abordera, sous forme de proposition de recherche et d'action, les objets d'interventions pertinents en vue d'agir sur la qualité de l'enseignement. Si l'on ne peut pas aborder concrètement la question de la qualité sans avoir recours à une évaluation, il faut en rendre explicites les fondements. Cela est d'autant plus nécessaire que la qualité de l'enseignement comporte de multiples dimensions, qu'il faut envisager dans leurs relations réciproques. Notre proposition de recherche comporte l'exploration de quelques dimensions de la notion de qualité, dans leur interrelation. Cette exploration devrait per-

\footnotetext{
*Communication présentée à la conférence annuelle de la Société canadienne pour l'étude de l'Enseignement Supérieur, Québec, juin 1976.
}

**Directeur du Bureau d'études, Université du Québec à Montréal. 
mettre de dégager des catégories susceptibles de recouvrir les principales caractéristiques identifiables de la notion de qualité, et qui doivent lui être substituées au cours de l'analyse et de l'action si l'on veut élaborer un processus opératoire d'évaluation de la qualité. Provisoirement, les catégories que nous suggérons sont les suivantes: l'environnement institutionnel, la relation enseignement-apprentissage, la dimension épistémologique de l'activité.

On trouvera en fin de texte un choix bibliographique concernant le thème de la qualité de l'enseignement universitaire.

\begin{abstract}
The Quality of University Teaching: Testing a Systematic Approach

The quality of university teaching is a much debated issue nowadays. Nevertheless, it seems that the more one hears about quality in university teaching, the less one knows what quality really means, how it can be appraised, and how one goes about improving it.

This paper presents some of the many different perspectives from which quality is viewed and, consequently, the contradictory uses to which so vague a concept is apt to be put. The need for a systematic approach to the quality of university teaching, which would take into account if not reconcile different points of view, is suggested. It is also suggested that it seems necessary, in view of the changes which take hold of the university environment, that higher education institutions experiment with and introduce tangible internal processes of quality evaluation.

As an example of the changes taking place, the paper describes the general evolution of the Quebec higher education system, in terms of student numbers and of costs, and the procedural changes in the process of approving and financing new programs of study.

The next section examines the obstacles - conceptual, analytical and operational related to quality evaluation and control.

Finally, a research proposal is outlined, which identifies three general research directions related to quality in university teaching: the institutionnal milieu and environment, the teaching-learning relationship, the epistemological aspects of the teaching activity. The basic assumption of this proposal is that only careful observation over time of these aspects in the institutionnal context is likely to conduce to the elaboration of relevant quality indicators.
\end{abstract}

Le contexte du débat sur la qualité

Engager le débat sur la qualité de l'enseignement universitaire de façon générale revient à multiplier des questions apparemment insolubles: qu'est-ce qu'une activité d'enseignement de qualité? a quoi se reconnait-elle? comment la comparer à des activités accomplies par des enseignants différents et destinées à des étudiants différents, dans un même domaine ou dans des domaines différents? comment évaluer globalement la qualité des programmes d'études qu'offre une institution, donc sa performance, et comment la comparer à la performance d'autres institutions? 
Inévitablement, une discussion amorcée en ces termes aboutit vite à des impasses. Pour s'en convaincre il suffit de rappeler quelques-uns des paradoxes auxquels fait face le système universitaire de nos jours. C'est au nom de la qualité de l'enseignement que les institutions réclament l'augmentation des ressources que le corps social met à leur disposition. C'est aussi au nom de la qualité des activités et de leur efficacité que les gouvernements et les corps législatifs intensifient les contrôles de la gestion des ressources universitaire. C'est également au nom de la qualité des activités que les professeurs des universités en sont venus à se syndiquer et à négocier collectivement leurs conditions de travail. Enfin, c'est au nom de la même qualité des enseignements que les étudiants réclament et obtiennent de plus en plus de pouvoirs dans le contrôle des programmes qu'ils poursuivent. Faut-il ajouter, dans ces conditions, que la notion de "qualité de l'enseignement" est passablement élastique, qu'elle peut signifier, selon les circonstances et selon celui qui l'invoque, une chose et son contraire, que les opinions divergent quant à ses fondements, en un mot, qu'il s'agit d'une notion subjective, qui implique des jugements de valeur?

Malgré tout, après avoir fait la part entre la rhétorique et les problèmes réels, après avoir élucidé les bases sur lesquelles reposeront les jugements de valeur, il semble nécessaire, voire indispensable d'entreprendre cet exercice au sein des universités: l'évolution même du système universitaire et la période d'incertitude qu'il traverse y invitent de manière pressante.

Il n'y a pas si longtemps, la qualité de l'enseignement dans une université était une chose qui semblait aller de soi. Elle était en quelque sorte inhérente à l'entreprise elle-même, du moins telle qu'elle était vue de l'extérieur. Le langage courant lui-même témoigne de cette similitude ou, selon les points de vue, de cette confusion, qui tolérait un usage synonymique des notions "enseignement supérieur" et "université". Peut-être la tendance à utiliser de plus en plus fréquemment le terme "enseignement post-secondaire", afin de désigner le niveau qui va des études collégiales aux études post-doctorales signale-t-elle la fin des illusions? La qualité n'est plus considérée comme un attribut automatique de l'enseignement universitaire mais plutôt comme un objectif à atteindre, un élément qu'il faut produire, cultiver et améliorer constamment.

Bien sûr, tant en Amérique du Nord qu'en Europe, il y a eu depuis toujours des universités plus prestigieuses que d'autres, des institutions dont la renommée universelle dans certains champs de la connaissance a contribué à leur conférer, par un effet d'halo, un prestige dans d'autres domaines. Mais ce n'est que depuis les années 60, avec l'avènement de l'enseignement supérieur de masse aux Etats-Unis et la prolifération correspondante des programmes que le problème de la qualité a commencé à se poser de façon plus aiguë. L'essai de Cartter $(8)^{*}$ sur la classification des programmes de 2 e et $3 \mathrm{e}$ cycles aux Etats-Unis, en fonction de leur qualité, date de 1966. Fait curieux mais explicable, pendant la période de grande expansion des années 60 , la question de la qualité a fait l'objet de peu de recherches. Depuis que l'éthique de l'expansion a commencé à céder le pas aux impératifs d'une croissance au ralenti, on observe un regain d'intérêt général pour l'aspect qualitatif des activités universitaires.

Il va sans dire, les facteurs déterminants dans cette évolution ont été les transformations rapides qu'a subies le système universitaire depuis quinze ans. Comme dans un mouvement

*Les chiffres entre parenthèses renvoient aux références bibliographiques. 
de balancier, l'augmentation sans précédent des effectifs étudiants et des coûts d'opération des universités entre 1960 et 1970 a été suivie d'une période où l'expansion du système universitaire s'est nettement ralentie. On craint même que ce ralentissement ne se transforme en une contraction absolue du volume d'activités, due notamment à la réduction de la proportion des jeunes qui poursuivent des études universitaires. Quoi qu'il en soit, le ralentissement de l'expansion et les difficultés qui l'accompagnent ont imposé aux institutions universitaires de porter leur attention sur un thème relativement nouveau, celui de la "croissance qualitative", c'est-à-dire du renforcement du processus éducatif, de l'adapta-

Tableau 1

Effectifs d'étudiants et subventions aux universités du Québec: 1962 à 1975

\begin{tabular}{|c|c|c|c|c|}
\hline & Effectifs d'étudiants* & $\frac{\text { Variation }}{(\%)}$ & $\frac{\text { Subvention }}{\text { (\$ millions) }}$ & $\frac{\text { Variation }}{(\%)}$ \\
\hline $1962-63$ & $24,631^{*}$ & & 20 & \\
\hline $1963-64$ & 26,994 & 9.2 & 22 & 10.0 \\
\hline $1964-65$ & 30,820 & 14.2 & 27 & 22.7 \\
\hline $1965-66$ & 34,788 & 12.9 & 35 & 29.6 \\
\hline $1966-67$ & 38,164 & 9.7 & 44 & 25.7 \\
\hline $1967-68$ & 43,525 & 14.0 & 67 & 52.3 \\
\hline $1968-69$ & 47,588 & 9.3 & 85 & 26.9 \\
\hline $1969-70$ & 56,008 & 17.7 & 122 & 43.5 \\
\hline $1970-71$ & $66,993^{*}$ & & 143 & 17.2 \\
\hline $1971-72$ & 69,082 & 3.1 & 177.1 & 21.7 \\
\hline 1972.73 & 75,607 & 9.4 & 207.8 & 14.8 \\
\hline $1973-74$ & 79,531 & 5.2 & 231.7 & 11.5 \\
\hline $1974-75$ & 90,397 & 13.7 & 265.7 & 14.7 \\
\hline
\end{tabular}

*A temps complet jusqu'en 1969-70; équivalents à plein temps depuis 1970-71

\section{Sources:}

- Objectifs généraux de l'enseignement supérieur et grandes orientations des établissements, Cahier I, L'évolution récente de l'enseignement supérieur au Québec, Conseil des Universités, juillet 1972 .

- Population étudiante des universites du Québec. Statistiques 1962-1982. DGES, s.d.

- $3^{e}$ Rapport annuel 1971/72, Conseil des universités, août 1972

- $5^{e}$ Rapport annuel 1973/74, Conseil des universités, août 1974

- $\sigma^{e}$ Rapport annuel 1974/75, Conseil des universités, août 1975. 
tion à de nouveaux besoins sociaux et à de nouveaux publics, de l'utilisation efficace de ressources limitées (9).

Le tableau suivant indique l'évolution générale des effectifs d'étudiants et des subventions aux universités du Québec, entre 1962 et 1975.

On peut constater donc que, si depuis 1962 l'effectif d'étudiants s'est multiplié environ par quatre, les subventions se sont multipliées environ par treize. En même temps que cela marque le rôle prépondérant que joue l'Etat aujourd'hui dans le financement des universités, cela explique son souci de tempérer l'augmentation des coûts: entre 1962 et 1973 les subventions ont décuplé alors qu'en 1975 les sommes consacrées à l'enseignement supérieur ne représentaient qu'un peu plus du double de la subvention de 1970.

Récemment on a remarqué toutefois non seulement un rythme plus lent de croissance des subventions, mais une diminution des ressources réelles par étudiant:

\section{Tableau 2}

Taux de variation des effectifs étudiants et des ressources réelles par étudiant: $1971-72$ à $1974-75$

\begin{tabular}{ccccc} 
& \multicolumn{2}{c}{ Effectifs d'étudiants } & & \multicolumn{2}{c}{ Ressources réelles par étudiant } \\
\cline { 2 - 3 } $1971-72$ & 4.1 & & $\frac{\text { Maximum* }}{2.0}$ & Minimum* \\
$1972-73$ & 5.5 & & 3.3 & 3.2 \\
$1973-74$ & 5.1 & & -2.7 & 1.4 \\
$1974-75$ & $2.3^{* *}$ & & $2.4^{* *}$ & -4.5 \\
\hline
\end{tabular}

**Selon que l'augmentation de l'âge moyen (coûts imputables au vieillissement du personnel) est considérée ou non comme facteur de productivité.

**estimation à réviser à la lumière des effectifs réels.

Source:

Cinquième rapport annuel, Conseil des universités, août 1975.

Qu'il s'agisse là d'un phénomène isolé ou d'un signal qui indique une tendance à plus long terme, cet état de choses a contribué à ce que les universités abordent de façon directe la question de la qualité de l'enseignement. Ainsi, selon le Conseil des universités, "c'est à l'unanimité que les universités réagissent aux contraintes de l'enveloppe des subventions et indiquent leur inquiétude quant à l'évolution générale de la qualité de l'enseignement supérieur. Une inquiétude si généralisée ne peut être ignorée ou passée sous silence; car même si la qualité de l'enseignement n'est pas uniquement fonction de fluctuations dans les ressources, on ne peut nier qu'une diminution importante des ressources disponibles 
est un indicateur de baisse de qualité qui ne doit pas être négligé" (13). C'est dans ce contexte que le Conseil des universités recommande de mettre au point des indicateurs de qualité qui permettent d'évaluer l'enseignement supérieur.

Il existe une autre raison, à la fois plus immédiate et plus fondamentale, qui devrait amener les universités du Québec à se préoccuper davantage de l'évaluation de leurs activités d'enseignement. Désormais, le Comité conjoint des programmes, mandaté par le Ministère de l'Education et par le Conseil des universités afin de coordonner les programmes d'études, laissera aux universités elle-mêmes la responsabilité d'analyser la qualité de leurs projets de nouveaux programmes, en se réservant le droit de statuer uniquement sur les projets dont il aura établi l'opportunité du point de vue de l'ensemble du système d'enseignement supérieur. Seuls ces derniers projets bénificieront, si la qualité en est établie, d'un financement particulier. Il en ressort que, en dehors de ces cas somme toute exceptionnels, les universités devront financer à même leurs ressources régulières les projets dont la réalisation semble indispensable à leur développement institutionnel.

C'est là la perspective de choix difficiles et d'arbitrages extêmement serrés dans l'allocation des ressources d'une institution. Dans cette conjoncture, la question de la qualité des activités d'enseignement et des instruments susceptibles de l'évaluer revêt une importance décisive pour le devenir des universités.

\section{Les difficultés inhérentes à l'évaluation de la qualité}

La plupart des recherches récentes ayant pour but d'évaluer diverses composantes de l'enseignement supérieur soulignent les obstacles conceptuels et opératoires que rencontre inévitablement l'effort d'apprécier les effets de l'enseignement supérieur.

Qu'il s'agisse de l'évaluation des institutions en vertu du principe de leur responsabilité envers le corps social pourvoyeur de fonds $(4,12,18)$, qu'il s'agisse de l'effort d'estimer et d'améliorer la productivité des universités (29), qu'il s'agisse d'estimer l'impact général de l'enseignement supérieur $(1,3,11,20)$ ou encore de juger de la qualité des institutions et de leurs programmes $(2,5,8,21,27)$, les difficultés rencontrées sont de deux ordres. Les unes peuvent être considérées comme étant inhérentes au processus d'éducation lui-même, dont les "produits", tout en étant impalpables, ont des effets tant sur l'individu que sur la société dans son ensemble. Ce sont là, essentiellement des difficultés de définition et de "mesure", donc de quantification de la qualité (la contradiction dans les termes en est d'ailleurs révélatrice). Cette série de difficultés n'est sans doute pas étrangère à la diversité des aspects et des intervenants, partant à la multiplicité des angles sous lesquels il est possible d'aborder le problème de la qualité (v. 111).

Une autre série de difficultés apparaît lorsqu'on essaie d'aborder concrètement ce problème au sein des universités et d'y introduire des processus d'évaluation en vue d'améliorer la qualité des activités d'enseignement. Ces difficultés ont pour origine la nature conflictuelle des relations qui existent entre diverses composantes de l'institution universitaire, lorsqu'il n'y a plus de consensus sur les objectifs, les priorités, la forme de gouvernement et de prise de décisions ou même la légitimité de certains groupes ou actions. C'est là le cas d'institutions où le gouvernement par consensus ou par autorité partagée est remplacé par des relations régies par le Code du travail, donc par l'arbitrage d'un contentieux permanent. Dans ces conditions, l'introduction de processus d'évaluation de la qualité rencontrera sans doute des difficultés d'ordre opératoire. 


\section{Problèmes de définition et de mesure}

On l'a souligné précédemment, la difficulté conceptuelle d'évaluer la qualité de l'enseignement et de ses résultats tient à la nature même du processus éducatif et à la diversité des valeurs qui s'y rattachent. Pour savoir si les "résultats" du système d'enseignement supérieur sont à la hauteur des investissements social et individuel qu'on y consacre, il faudrait que ces deux dimensions soient directement commensurables ou qu'elles soient convertibles en une unité de mesure acceptable. De toute évidence ce n'est pas le cas, du moins pour certains résultats intangibles de l'enseignement supérieur, $\dagger$ tels ses effets sociaux: accroissement et diffusion du savoir et du bien-être, ou des conséquences négatives qui en résultent, socialisation, préservation et enrichissement de l'héritage culturel, critique et changements sociaux. Malgré les controverses qui entourent ces "résultats" de l'enseignement supérieur et leur degré d'utilité, ils devraient être pris en considération lorsqu'on prétend établir un rapport "coûts-bénéfices" et estimer si des investissements alternatifs (dans le secteur économique, ou en santé, par exemple) seraient socialement plus rentables.

Or il n'existe pas aujourd'hui et ce, à quel que niveau que ce soit, y compris au niveau de décision gouvernemental, des instruments d'analyse qui permettent d'éclairer sinon de fonder ce type de décision, si ce n'est par choix d'ordre politique. Comme le souligne Bowen (4), cette situation n'a rien d'extraordinaire si on la compare a des centaines d'autres domaines où l'action humaine est fondée sur des évaluations et des décisions intuitives. Par ailleurs, non seulement cette situation ne devrait-elle pas empêcher de fonder l'évalu. ation de la performance et de la qualité sur des données et sur des faits aussi objectifs que possible, même s'ils ne sont pas toujours quantifiables, mais elle devrait stimuler le recensement et l'analyse systématiques de ces faits.

La plupart des chercheurs sérieux mettent en garde contre les interprétations hâtives que l'on pourrait tirer quant à la performance et à la qualité sur la foi des seules données quantifiables: rapports professeurs-étudiants, budgets de fonctionnement, coûts par étudiant, nombre des volumes des bibliothèques, pourcentage des diplômes par rapport aux effectifs d'étudiants admis, nombre des publications, etc. $(4,15,17)$. En effet, étant donné les difficultés d'identifier et de mesurer les effets durables de l'enseignement supérieur, il existe une pression très forte en vue d'assimiler des indices quantitatifs comme ceux mentionnés plus haut à des indicateurs de qualité, alors qu'ils n'expriment que l'efficacité relative avec laquelle les institutions transforment des "intrants" (le temps des étudiants et des professeurs, les ressources financières et matérielles) en des "extrants" (effectifs, nombre de diplômés, nombre de publications, services divers au public, etc.). Contrairement à une opinion assez répandue, ce ne sont pas là des indicateurs de qualité proprement dits, mais des substituts qui permettent d'apprécier, très imparfaitement sans doute, comment les universités s'acquittent de leurs responsabilités en matière d'enseignement et de recherche, en relation avec les ressources dont elles disposent et d'estimer en quelque sorte leur productivité. Il est à noter donc qu'en rapportant la quantité des "extrants" à celle des "intrant" on ne porte pas de jugement sur la qualité des processus de transformation. Tout au plus pourrait-on essayer d'établir des rapprochements interinstitutionnels afin de voir si, dans des conditions similaires, leur performance est comparable selon certaines variables (par

$\dagger$ Par contre, des recherches économiques ont démontré que linvestissement individuel dans l'éducation universitaire comporte un taux de rentabilité comparable à celui des ressources financières, dans une économie de marché de type occidental. (29) 
exemple, capacité des diplômés de trouver et garder un emploi, proportion des diplômés qui poursuivent leurs études, temps moyen écoulé entre l'admission et l'obtention du diplôme, moyenne des heures de contact avec les étudiants par professeur équivalent à temps plein, etc.)

\section{Problèmes opératoires}

A la différence de la catégorie précédente, les obstacles d'ordre opératoire que rencontre l'effort d'évaluer la qualité de l'enseignement universitaire dépendent des caractéristiques institutionnelles. La tradition que représente une université, les valeurs particulières qu'elle véhicule, la forme de gouvernement et les relations qui s'établissent entre divers segments de la "communauté" universitaire, les modes particulières d'organisation des activités d'enseignement sont autant de facteurs susceptibles d'influencer la mise en oeuvre d'un processus d'évaluation de la qualité.

Pour donner un exemple, dans le cas de l'UQAM, il semble nécessaire de prendre en considération plusieurs caractéristiques qui lui sont propres, dont le mode d'organisation de l'enseignement et de la recherche, ainsi que les responsabilités particulières qui en découlent en matière d'évaluation des différentes composantes du système d'enseignement. Ainsi à l'UQAM, par règlement, les départements participent au processus d'évaluation des professeurs alors que c'est aux modules que revient la responsabilité d'organiser l'évaluation, par les étudiants, des enseignements de premier cycle. L'absence de mécanisme analogue au $2 \mathrm{e}$ cycle constitue un problème dans la mesure où l'on voudrait aboutir à un processus d'évaluation uniforme des enseignements par les étudiants, quel qu'en soit le niveau. Il va sans dire que l'évaluation effectuée par les étudiants n'est pas sans poser des problèmes de divers ordres $(29,39)$ sur lesquels nous ne nous attarderons pas ici, mais qui méritent de faire l'objet d'un examen en soi. Par ailleurs, ce partage étanche des responsabilités en matière d'évaluation, selon l'objet de l'évaluation, risque de rendre plus ardue la tâche d'introduire un système global d'évaluation de la qualité, qui prenne en ligne de compte les points de vue des étudiants, des professeurs et ceux de l'institution dans leur interrelation.

Pour résumer donc cette section, l'évaluation de la qualité des activités et des programmes d'enseignement d'une université pose tant des problèmes de réalisation que de sérieux problèmes de définition et de mesure. Il est néanmoins possible de construire certains indicateurs quantitatifs susceptibles d'être utilisés, de pair avec des formes d'évaluation de nature intuitive, comme approximations de la performance d'une institution. Il est nécessaire de souligner à nouveau cependant que ces indices fourniront davantage un aperçu des intrants que de la qualité des extrants ou de la qualité du processus éducatif lui-même, dont les effets véritables consistent en des changements individuels et sociaux à plus long terme.

\section{Une proposition de recherche d'indicateurs de la qualité}

Dans les pages qui précèdent nous avons montré que l'on ne peut pas aborder concrètement le problème de la qualité sans recourir à une évaluation. Comme les fondements peuvent en être multiples, il est nécessaire de les rendre explicites. Une première question est celle du.point de vue selon lequel on évalue les activités d'enseignement et des conditions dans 
lesquelles l'évaluation se déroule. Ainsi il n'est pas indifférent si le processus d'évaluation est contrôlé exclusivement par un seul groupe d'intérêts, soit-il les professeurs, les étudiants ou les administrateurs, s'il ne porte que sur certains aspects du processus éducatif à l'exclusion d'autres aspects, ou s'il constitue au contraire un processus cohérent et global, où l'on prend en considération l'ensemble des points de vue et où l'on aborde la totalité des aspects essentiels du système d'enseignement $(5,18,22)$.

L'objectif ultime de l'évaluation est d'améliorer la qualité des activités. Aussi faut-il, pour estimer si la qualité s'améliore, se reporter non seulement à des standards, qui peuvent varier eux-aussi selon la prépondérance accordée à l'un où l'autre des aspects impliqués, mais également à la qualité initiale des activités. En d'autres termes, la qualité est une notion relative.

Il faudrait donc, pour être exact, remplacer les références abstraites à la qualité de l'enseignement conçue comme une valeur intrinsèque par une recherche des déterminants de la qualité, c'est-à-dire de caractéristiques identifiables, susceptibles de lui être substituées au cours de l'analyse et de l'action en vue d'apprécier et d'améliorer la qualité des activités d'enseignement.

Ces caractéristiques identifiables, nous proposons de les rechercher le long des axes suivants:

- les ressources et l'environnement institutionnels

- la relation enseignement-apprentissage

- les aspects épistémologiques de l'activité

Ce sont là des catégories qui recouvrent les principaux aspects de la notion de qualité ou, si l'on veut, les points de vue selon lesquels on peut aborder ce problème, à savoir:

- le système d'enseignement

- le point de vue du professeur

- le point de vue de l'étudiant

- le marché du travail et les professions à accès limité

- la responsabilité institutionnelle en matière de qualité

La plupart de ces aspects ont fait l'objet de recherches plus ou moins poussées, dont on trouvera une sélection dans les références. Même un passage en revue rapide de la littérature convainc de la complexité du sujet et des difficultés d'agir dans ce domaine. Les groupes mandatés pour étudier cette question devraient donc concevoir leur tâche comme un travail de longue haleine et choisir parmi les multiples facettes de la notion de qualité, celles qu'ils voudraient explorer d'abord, ou même déterminer le point de vue ou l'ensemble des points de vue selon lesquels ils aborderont le problème. Faut-il envisager la question de l'évaluation de la qualité selon les objets d'évaluation (programmes, enseignements, etc.), ou encore selon les intervenants (responsabilité des professeurs, responsabilité des étudiants, responsabilité institutionnelle), ou encore faut-il considérer la question de la qualité de façon plus systématique?

Quelles que soient les réponses à ces questions, il serait possible d'entreprendre deux sortes d'actions:

- effectuer une recherche systématique sur l'un, l'autre ou l'ensemble des aspects qualitatifs des activités d'enseignement et sur ses ingrédients, en vue de se doter d'instruments d'analyse. 
- recueillir, dans un contexte donné, les informations nécessaires afin d'être en mesure de suggérer quelques indices susceptibles de révéler la présence, l'absence de ces ingrédients ou les changements qui interviennent dans leur dosage.

Ce n'est qu'après que ces deux séries d'actions auront donné quelques résultats, que l'on pourra aborder la mise en oeuvre de processus institutionnels de contrôle de la qualité de l'enseignement.

\section{Références}

1. Astin, Alexander W., "The Measured Effects of Higher Education," Annals of the American Academy of Political and Social Science, November, 1972.

2. Astin, Alexander W., "Undergraduate Achievement and Institutional 'Excellence'," Science, v. 161. August 1968.

3. Berls, Robert, "Exploration of the Determinants of Effectiveness in Higher Education." The Economics and Financing of Higher Education. U.S. Government Printing Office. Washington, D.C., 1969.

4. Bowen, Howard R., Evaluating Institutions for Accountability. Jossey-Bass, San Francisco, New Directions for Institutional Research, 1, 1974.

5. Cameron, John R., Measuring the Quality of Higher Education, Stoa, II.I, 1972, p. 5-13.

6. Carnegie Commission on Higher Education. Reform on Campus. Changing Academic Programs, A Report and Recommendations, June 1972. McGraw-Hill Book Co.

7. Carter, Charles F., "The Efficiency of Universities," Higher Education. Elsevier Publ. Co. 1(1972), p. $77-90$.

8. Cartter, Allan M., An Assesment of Quality in Graduate Education, Washington, D.C., American Council on Education, 1966.

9. Cartter, Allan M., ed., Assuring Academic Progress without Growth. Jossey-Bass, San Francisco, New Directions for Institutionnal Research, no. 6, 1975.

10. Centra, John A., Strategies for Improving College Teaching, AAHE, ERIC/HE Research Report no. 8, 1972.

11. Chickering, Arthur, "The Best Colleges have the least Effect." Saturday Review, January 16, 1971.

12. Conseil des universites, Cinquième rapport annuel 1973/74, Québec, août 1974.

13. Conseil des universites, Sixième rapport annuel 1974/75. Québec, août 1975.

14. Cross, Patricia K., "The New Learners," Change. February, 1973.

15. Dressel, Paul L. et. al., The Confidence Crisis. Jossey-Bass, San Francisco, 1971.

16. Eble, Kenneth E., Professors as Teachers. Jossey-Bass, San Francisco, 1973.

17. Elton, Charles E., Rodgers, Sam A.. "The Departmental Rating Game: Measure of Quantity or Quality?" Higher Education, (Amsterdam), 2(1973), p. 439-446.

18. Good, Harold M., Trotter, Bernard, "Accountability for Effective and Efficient University Teaching." Stoa, La revue canadienne d'enseignement supérieur, IV.1, 1974, p. 43-49.

19. Group for Human Development in Higher Education, Faculty Development in a Time of Retrenchment, Change Magazine, 1974.

20. Hodgkinson, Harold. "How Can We Measure the 'Value added' to Students by College?", Chronicle of Higher Education, Nov. 13, 1972.

21. Johnson, Henry, Rhodes, Dent M., Rumery, Robert E., The Assesment of Teaching in Higher Education: A Critical Retrospect and a Proposal. Higher Education, (Amsterdam), 4(1975), 173-199, 273-303.

22. Leibu, Ygal, Eléments pour étudier la problématique des modifications de programmes universitaires, UQAM, Bureau d'études, sept. 1973. 
23. Lewis, Brian M., "Educational Technology at the Open University: An Approach to the Problem of Quality," British Journal of Educational Technology, vo. 3 no. 3, 1972.

24. Mayhew, Louis B., Ford, Patrick J., Changing the Curriculum, Jossey-Bass, San Francisco, 1973.

25. Miller, Richard I., Evaluating Faculty Performance, Jossey-Bass, San Francisco, 1972.

26. Miller, Richard I., Developing Programs for Faculty Evaluation, A Source Book for Higher Education, Jossey-Bass, San Francisco, 1974.

27. Roose, K.D., Anderson, C.S., A Rating of Graduate Programs. Washington, D.C., American Council on Education, 1970.

28. Sheehan, Daniel S., "On the Invalidity of Student Ratings for Administrative Personnel Decisions," The Journal of Higher Education. XLVI, 6, 687-700.

29. Walhaus, Robert A., ed., Measuring and Increasing Academic Productivity, Jossey-Bass, San Francisco, New Directions for Institutional Research, no. 8, 1975.

30. Zelby, Leon W., "Student-Faculty Evaluation," Science, v. 183, 1267-1270. 